\title{
ARTICLE
}

\section{Atherogenic indices in pseudoexfoliation syndrome}

\author{
Enver Mirza $\mathbb{1}^{1}$
}

Received: 7 March 2019 / Revised: 14 May 2019 / Accepted: 22 May 2019 / Published online: 5 July 2019

(c) The Author(s), under exclusive licence to The Royal College of Ophthalmologists 2019

\begin{abstract}
Objectives Evaluation of atherogenic indices in patients with pseudoexfoliation syndrome (PEXS) by traditional serum lipid profiles [total cholesterol (TC), triglyceride (TG), low-density lipoprotein cholesterol (LDL-c), high-density lipoprotein cholesterol (HDL-c) and non-HDL-c] and non-traditional serum lipid ratios [TC/HDL-c, TG/HDL-c, LDL-c/HDL-c and non-HDL-c/HDL-c].

Methods A total of 100 patients were included in the study. Fifty patients diagnosed with PEXS were regarded as group 1 and 50 patients without PEXS were regarded as group 2, respectively.

Results The median TC, TG, LDL-c, HDL-c and non-HDL-c values were significantly higher in group 1 compared to group 2 ( $p=0.007, p=0.025, p=0.016, p=0.015$ and $p=0.042$, respectively). But there were no significant differences in the TC/HDL-c, TG/HDL-c, LDL-c/HDL-c and non-HDL-c/HDL-c ratios among the two groups $(p=0.581, p=0.617$, $p=0.292$ and $p=0.583$, respectively).

Conclusions Non-traditional serum lipid ratios are superior to traditional serum lipid profiles for identifying the risk of vascular disease and this study did not demonstrate a complete relationship between PEXS and increased risk of vascular disease.
\end{abstract}

\section{Introduction}

Pseudoexfoliation syndrome (PEXS), is a well-recognised but not well-understood disorder that primarily observed as an ocular manifestation. This syndrome is characterised by the accumulation of a typical fibrillary material in the anterior segment of the eyes which is frequently associated with some ocular complications such as cataract, zonular disruption, glaucoma, and retinal vein occlusion [1]. It is known that pseudoexfoliation (PEX) material is produced by the corneal endothelium, trabecular endothelium, iris pigment epithelium, non-pigmented ciliary epithelium and lens epithelium [1].

As far as is known, this is an age-related and multifactorial (genetic and environmental factors) syndrome that affects more than 70 million people worldwide [2]. Previous studies indicated that trauma, infections,

Enver Mirza

envermirza@gmail.com

1 Department of Ophthalmology, University of Health Sciences, Konya Education and Research Hospital, 42090 Konya Meram, Turkey ultraviolet radiation, endothelial dysfunction, oxidative stress and increased ocular inflammation play key roles in the pathogenesis of PEXS [1, 3, 4].

PEX material can be easily diagnosed with slit-lamp biomicroscopic examination by ophthalmologists due to the visualisation of this white-grey extracellular material in the pupillary margin or anterior lens capsule. Moreover, PEX material has been observed in the heart, vessels, liver, kidney, lung, cerebral meninges and skin [5-7]. These observations brought an idea to the minds that PEXS may be a systemic disorder. Indeed, the association between PEXS and many systemic diseases were evaluated in the literature [8]. In these studies, there is a general consensus regarding the relationship between PEXS and increased risk of vascular diseases $[9,10]$. Especially, PEX is declared as an independent risk factor for cardiovascular diseases [11, 12]. In addition, the presence of dyslipidemia increases the incidence of systemic vascular diseases, causes endothelial dysfunction and decreases antioxidant defence [13, 14].

On this basis, it was aimed in this study to investigate the risk of vascular disease and the relationship between serum lipid parameters and lipid ratios in patients with PEXS. 


\section{Materials and methods}

This retrospective study comprised patients undergoing phacoemulsification surgery due to senile cataract between March 2017 and October 2018 at the Department of Ophthalmology, Konya Education and Research Hospital, University of Health Sciences. All phacoemulsification surgeries were done by the same surgeon (EM). This study was approved by the local ethical committee (No: 2018/1578) and was conducted with adherence to the tenets of the Helsinki declaration. All patients underwent a comprehensive ophthalmologic examination including bestcorrected visual acuity (BCVA), intraocular pressure measurement by Goldmann applanation tonometry, anterior segment examination, and dilated fundus examination by the slit-lamp biomicroscope. The diagnose of cataract based on due to the presence of opacity in the crystalline lens and the diagnose of PEXS based on due to the visualisation of this white-grey PEX material in the pupillary margin or anterior lens capsule after dilation by slit-lamp biomicroscopic examination.

A total of 100 patients diagnosed with cataract were reviewed and included in the study. The patients were classified into two groups. Fifty patients ( 24 female and 26 male) with PEXS considered as group 1 and 50 patients (25 female and 25 male) without PEXS considered as group 2. The demographic data and ocular examination findings, blood parameters of participants were accessed in the file records and laboratory archives of Konya Education and Research Hospital, University of Health Sciences. Routine blood samples were drawn after a fasting period of $8-12 \mathrm{~h}$ from all patients before surgery. Total cholesterol (TC), triglyceride (TG), high-density lipoprotein cholesterol (HDL-c) and low-density lipoprotein cholesterol (LDL-c) levels values were evaluated by the chemistry analyser (Beckman Coulter AU5800, USA). All patients underwent blood testing for hepatic function and renal function to determine final inclusion; patients with liver enzymes $>3$ times the normal reference values or with creatinine $>130 \mathrm{mmol} / \mathrm{L}$ were excluded from the study. Also, the patients with any other ocular diseases except cataract and PEXS were not included in the study. Current treatment with any systemic medications which may affect blood parameters especially steroid or antihyperlipidaemia therapy, history of malignancy, acute systemic and local infections, acute or chronic liver and kidney diseases, autoimmune diseases, smoking, and alcohol consumption are the criteria of exclusion. Moreover, TC to HDL-c ratio (TC/HDL-c), TG to HDL-c ratio (TG/HDL-c), LDL-c to HDL-c ratio (LDL-c/HDL-c), non-HDL-c to HDL-c ratio (non-HDL-c/HDL-c) and non-HDL-c values were calculated. TG/HDL-c was regarded as the index of insulin resistance [15].
TC/HDL-c, LDL-c/HDL-c, non-HDL-c/HDL-c and nonHDL-c were regarded as an atherogenic index [15-20].

All statistical analyses of this study were performed with SPSS for Windows 22.0 package programme (SPSS Inc., Chicago, IL, USA). Data were expressed as medians with the interquartile range (25th-75th percentiles) according to the distributions of the continuous variables. Pearson's $\chi^{2}$ test was performed for categorical data analysis. The Kolmogorov-Smirnov test was used to test normality of distribution. Comparisons of parametric values among groups were performed by one-way ANOVA. Comparisons of non-parametric values among groups were performed by the Kruskal-Wallis test. $p<0.05$ was considered statistically significant.

\section{Results}

The median ages were 71.5 years $(Q 1=66.5 ; Q 3=80.5)$ in group 1 and 73 years $(Q 1=62 ; Q 3=78.25)$ in group 2 . Demographic characteristics of all the patients are shown in Table 1. There were no statistical differences in age and gender between the groups $(p=0.34$ and $p=0.841)$. There were no significant differences in the groups, according to the presence of diabetes mellitus, hypertension, and cerebrovascular diseases $(p=0.488, p=0.171$ and $p=0.749$, respectively) but cardiovascular diseases were significantly higher in group $1(p=0.021)$.

The median TC, TG, LDL-c, HDL-c and non-HDL-c values were significantly higher in group 1 compared to group 2 as shown in Table $2(p=0.007, p=0.025, p=$ $0.016, p=0.015$ and $p=0.042$, respectively). In addition, there were no significant differences in the TC/HDL-c, TG/ HDL-c, LDL-c/HDL-c and non-HDL-c/HDL-c ratios among the two groups $(p=0.581, p=0.617, p=0.292$ and $p=0.583$, respectively; Table 2 ).

\section{Discussion}

The pathophysiologic mechanism which is responsible for the development of PEXS remains unclear. But many researches are still ongoing to clarify this phenomenon. Investigating the associations between PEXS and other diseases may give us some clues to elucidate this disorder. Hence, increased vascular risk in PEXS has been the subject of many articles and previously many studies reported that there is a link between coronary artery diseases, ischaemic heart disease, hypertension, diabetes mellitus and PEXS [21-27]. It is mentioned that PEX is related to vascular wall abnormalities and may have a crucial role in the pathogenesis of arterial diseases [4, 7, 21]. Also, it is believed that dyslipidemia increases the risk of systemic vascular diseases due to decreasing antioxidant 
Table 1 The demographic data of the patients

\begin{tabular}{llll}
\hline & Group 1 (PEXS, $n=50)$ & Group 2 (non - PEXS, $n=50)$ & $P$-value \\
\hline Age [median $\left.\left(Q_{1}-Q_{3}\right)\right]$ & $71.5(66.5-80.5)$ & $73.00(62.00-78.25)$ & 0.341 \\
Gender $(n$, female/male) & $24 / 26$ & $25 / 25$ & 0.841 \\
Diabetes mellitus $(n)$ & 14 & 11 & 0.481 \\
Hypertension $(n)$ & 16 & 10 & 0.171 \\
Cardiovascular disease $(n)$ & 11 & 3 & 0.021 \\
Cerebrovascular disease $(n)$ & 5 & 6 & 0.749 \\
\hline
\end{tabular}

PEXS pseudoexfoliation syndrome

Statistically significant $P$ values are in Italic $<0.05$

\begin{tabular}{lccc}
\hline & \multicolumn{2}{l}{ Median $\left(Q_{1}-Q_{3}\right)$} & \\
\cline { 2 - 3 } & Group 1 $($ PEXS, $n=50)$ & Group 2 (non-PEXS, $n=50)$ & $P$-value \\
\hline TC $(\mathrm{mg} / \mathrm{dL})$ & $205.0(173.5-251.0)$ & $186.00(168.25-201.00)$ & 0.007 \\
TG $(\mathrm{mg} / \mathrm{dL})$ & $141.5(92.0-196.5)$ & $124.00(98.50-142.25)$ & 0.025 \\
LDL-c $(\mathrm{mg} / \mathrm{dL})$ & $124.0(102.0-163.5)$ & $113.0(98.8-130.0)$ & 0.016 \\
HDL-c $(\mathrm{mg} / \mathrm{dL})$ & $49.5(42.7-59.5)$ & $45.0(39.0-52.0)$ & 0.015 \\
Non-HDL-c $(\mathrm{mg} / \mathrm{dL})$ & $153.5(124.5-200.8)$ & $140.0(126.5-154.0)$ & 0.042 \\
TC/HDL-c & $4.2(3.4-4.9)$ & $4.1(3.7-4.7)$ & 0.581 \\
TG/HDL-c & $2.9(1.7-3.7)$ & $2.6(1.9-3.6)$ & 0.617 \\
LDL-c/HDL-c & $2.7(2.1-3.3)$ & $2.5(2.2-2.7)$ & 0.292 \\
non-HDL-c/HDL-c & $3.2(2.39-3.9)$ & $3.1(2.7-3.6)$ & 0.583 \\
\hline
\end{tabular}

PEXS pseudoexfoliation syndrome, $T C$ total cholesterol, $T G$ triglyceride, $L D L-c$ low-density lipoprotein cholesterol, $H D L-c$ high-density lipoprotein cholesterol

Statistically significant $P$ values are in Italic $<0.05$ defence and causing endothelial dysfunction [13, 14]. In the vascular calcification process serum levels of TC, TG and LDL-c were found to be elevated and serum HDL-c levels found to be low $[28,29]$.

Furthermore, arterial stiffness is a predictor factor for cardiovascular disease risk and it is known that serum HDLc has a protective effect on arterial stiffness [30, 31]. NonHDL-c is a measurement of the cholesterol in LDL-c and very low-density lipoprotein (VLDL-c) particles. In the previous studies, it was mentioned that non-HDL-c is a superior determinant than LDL-c for evaluating arterial stiffness [32, 33]. It may be proposed that there is a general opinion about these non-traditional serum lipid ratios (TC/ HDL-c, TG/HDL-c, LDL-c/HDL-c and non-HDL-c/HDL-c) are more discriminative for atherogenic events than other single traditional lipid profiles. It is reported that non-HDLc/HDL-c ratio is more powerful, easy and cost-effective to indicate coronary artery disease risk, arterial stiffness risk and insulin resistance than LDL-c, HDL-c and non-HDL-c in clinic practice [33-36]. Also, it is demonstrated that TC/HDL-c and the LDL-c/HDL-c ratios are better markers for indicating cardiovascular diseases and atherosclerosis risk [37-40]. TG/HDL-c ratio is considered as another predictive ratio than other single lipid profiles to predict cardiovascular diseases and insulin resistance [41-43].
Commonly, previous studies have focused on conventional serum lipids profiles in PEXS and the studies investigating the TC/HDL-c, TG/HDL-c and non-HDL-c values in PEXS are very less and to the best of my knowledge, there were no studies investigating the LDL-c/HDL-c and non-HDL-c/HDL-c values in PEXS [44, 45]. In the study of Türkyılmaz et al., TC, TG and LDL-c levels found to be significantly higher and HDL-c levels were lower in the PEX group (40 patients) [46]. Also, in the study of Kurtul et al., they found no differences in the levels of serum TC, TG and HDL-c among PEXS group (52 patients), pseudoexfoliation glaucoma (PEXG) group (20 patients) and control group. But LDL-c levels were significantly higher in the PEXS and PEXG groups [47]. However, there were no differences in the serum levels of TC, TG, HDL-c and LDL-c in the study of Atalar et al. (23 PEX patients), in the two studies of Lesiewska et al. (74 PEX patient, 96 PEX patients, respectively), and in the study of Speckauskas et al. (152 PEX patients) [4, 44, 45, 48]. In the two studies of Lesiewska et al., there were no significant differences in the TG/HDL-c, TC/HDL-c and non-HDL-c values between PEX group and healthy subjects $[44,45]$. But in the present study, all conventional serum lipids profiles, non-traditional serum lipid ratios additionally including LDL-c/HDL-c and non-HDL-c/HDL-c values were investigated and it was 
found that the levels of traditional serum lipid profiles and the levels of non-HDL-c were significantly higher in the PEXS group but there were no significant differences in the values of TG/HDL-c, TC/HDL-c, LDL-c/HDL-c and non-HDL-c/HDL-c between PEXS group and without PEXS group.

The samples size and designs of the published studies are different from each other. Thus, the results may be controversial. In addition, it has to be mentioned that dietary habits may influence lipid levels [49]. Hence, the discrepancy of the studies' results may be related to the dietary habits of the patients. Due to the retrospective design, this information could not be obtained from the present study groups. Therewithal, the small number size of patients and single-centre study design are the other limitations of this study.

\section{Conclusion}

The relationship between PEXS and increased risk of vascular disease has been shown in previous studies with different methods. The results of this study did not show a linkage between PEXS and the increased risk of vascular disease, completely. The serum lipid profiles were higher in patients with PEXS than in the controls, but it was not possible to mention a similar difference in the serum lipid ratios among the two groups. Due to these controversial results, further studies with larger sample size are required to explain this topic.

\section{Summary}

\section{What was known before}

- It is assumed that PEX is related to several vascular diseases. Therefore, many studies have investigated serum lipid profiles to elucidate this relationship but the results were controversial.

\section{What this study adds}

- The relationship between atherogenic indices and pseudoexfoliation syndrome was discussed in this study, which showed that non-traditional serum lipid ratios, which are superior to traditional serum lipid profiles, were not higher in PEXS than those of controls.

Acknowledgements The author would like to thank Adnan Karaibrahimoglu, MD for statistical assistance.

\section{Compliance with ethical standards}

Conflict of interest The authors declare that they have no conflict of interest.

Publisher's note: Springer Nature remains neutral with regard to jurisdictional claims in published maps and institutional affiliations.

\section{References}

1. Scharfenberg E, Schlötzer-Schrehardt U. PEX syndrome:clinical diagnosis and systemic manifestations. Ophthalmologe 2012;109: 952-61.

2. Nazarali S, Damji F, Damji KF. What have we learned about exfoliation syndrome since its discovery by John Lindberg 100 years ago? Br J Ophthalmol 2018;102:1342-50.

3. Sorkhabi R, Ghorbanihaghjo A, Ahoor MH. Oxidative stres in psudoexfoliation syndrome. Indian J Ophthalmol 2011;23: 27-32.

4. Atalar PT, Atalar E, Kilic H, Abbasoglu OE, Ozer N, Aksöyek S, et al. Impaired systemic endothelial function in patients with pseudoexfoliation syndrome. Int Heart J 2006;47:77-84.

5. Morrison JC, Green WR. Light microscopy of the exfoliation syndrome. Acta Ophthalmol 1988;184:5-27.

6. Schlotzer-Schrehardt UM, Koca MR, Naumann GO, Volkholz H. Pseudoexfoliation syndrome. Ocular manifestation of a systemic disorder? Arch Ophthalmol 1992;110:1752-6.

7. Schumacher S, Schlotzer-Schrehardt U, Martus P, Lang W, Naumann GO. Pseudoexfoliation syndrome and aneurysms of the abdominal aorta. Lancet 2001;357:359-60.

8. Andrikopoulos GK, Alexopoulos DK, Gartaganis SP. Pseudoexfoliation syndrome and cardiovascular diseases. World $\mathrm{J}$ Cardiol 2014;6:847-54.

9. Visontai Z, Merisch B, Kollai M, Hollo G. Increase of carotid artery stiffness and decrease of baroreflex sensitivity in exfoliation syndrome and glaucoma. Br J Ophthalmol 2006;90:563-7.

10. French DD, Margo CE, Harman LE. Ocular pseudoexfoliation and cardiovascular disease: a national cross-section comparison study. N Am J Med Sci 2012;4:468-73.

11. Wang W, He M, Zhou M, Zhang X. Ocular pseudoexfoliation syndrome and vascular disease: a systematic review and metaanalysis. PLoS One. 2014;9:e92767.

12. Katsi V, Pavlidis AN, Kallistratos MS, Fitsois A, Bratsas A, Tousoulis D, et al. Cardiovascular repercussions of the pseudoexfoliation syndrome. N Am J Med Sci 2013;5:454-9.

13. Di Angelantonio E, Sarwar N, Perry P, Kaptoge S, Ray KK. et al. Emerging Risk Factors Collaboration. Major lipids, apolipoproteins, and risk of vascular disease. J Am Med Assoc. 2009;302:1993-2000.

14. Sun GZ, Li Z, Guo L, Zhou Y, Yang HM, Sun YX. High prevalence of dyslipidemia and associated risk factors among rural Chinese adults. Lipids health Dis 2014;13:189.

15. Ren X, Chen ZA, Zheng S, Han T, Li Y, Liu W, et al. Association between triglyceride to HDL-C ratio (TG/HDL-C) and insulin resistance in Chinese patients with newly diagnosed type 2 diabetes mellitus. PLoS One 2016;11:e154345.

16. Nogay NH. Assessment of the correlation between the atherogenic index of plasma and cardiometabolic risk factors in children and adolescents: might it be superior to the TG/HDL-C ratio? J Pediatr Endocrinol Metab 2017;30:947-55.

17. Dobiasova M. Atherogenic impact of lecithin-cholesterol acyltransferase and its relation to cholesterol esterification rate in HDL 
(FER(HDL)) and AIP $[\log (\mathrm{TG} / \mathrm{HDL}-\mathrm{C})]$ biomarkers: the butterfly effect? Physiol Res 2017;66:193-203.

18. Kiyosue A. Nonfasting TG/HDL-C ratio seems a good predictor of MACE in CAD patients with statin therapy. Could it be a treatment target? J Cardiol 2018;71:8-9.

19. Fernandez ML, Webb D. The LDL to HDL cholesterol ratio as a valuable tool to evaluate coronary heart disease risk. J Am Coll Nutr. 2008;27:1-5

20. Arsenault BJ, Rana JS, Stroes ES, Despres JP, Shah PK, Kastelein $\mathrm{JJ}$, et al. Beyond low-density lipoprotein cholesterol: respective contributions of nonhigh-density lipoprotein cholesterol levels, triglycerides, and the total cholesterol/high-density lipoprotein cholesterol ratio to coronary heart disease risk in apparently healthy men and women. J Am Coll Cardiol. 2009;55:35-41.

21. Mitchell P, Wang JJ, Smith W. Association of pseudoexfoliation syndrome with increased vascular risk. Am J Ophthalmol 1997; 124:685-7.

22. Citirik M, Acaroglu G, Batman C, Yildiran L, Zilelioglu O. A possible link between the pseudoexfoliation syndrome and coronary artery disease. Eye 2007;21:11-5.

23. Speckauskas M, Tamosiunas A, Jasinskas V. Association of ocular pseudoexfoliation syndrome with ischaemic heart disease, arterial hypertension, and diabetes mellitus. Acta Ophthalmol 2012;90:470-5.

24. French DD, Margo CE, Harman LE. Ocular pseudoexfoliation and cardiovascular disease: a national cross-section comparison study. N Am J Med Sci 2012;4:468-73.

25. Yokusoglu M. Is pseudoexfoliation syndrome a risk factor for cardiovascular diseases? Anadolu Kardiyol Derg 2012;12:488-9.

26. Akdemir MO, Sayin MR, Armut M, Akpinar I, Ugurbas SH. Pseudoexfoliation syndrome and coronary artery ectasia. Eye 2014;28:594-9.

27. Hollo G. Exfoliation syndrome and systemic cardiovascular diseases. J Glaucoma 2014;23:9-11.

28. McCullough PA. Effect of lipid modification on progression of coronary calcification. J Am Soc Nephrol 2005;16:115-9.

29. Penalva RA, Huoya Mde O, Correia LC, Feitosa GS, Ladeia AM. Lipid profile and intensity of atherosclerosis disease in acute coronary syndrome. Arq Bras Cardiol. 2008;90:24-30.

30. Mattace-Raso FU, van der Cammen TJ, Hofman A, et al. Arterial stiffness and risk of coronary heart disease and stroke: the Rotterdam Study. Circulation 2006;113:657-63.

31. Cohn JN. Arterial stiffness, vascular disease, and risk of cardiovascular events. Circulation 2006;113:601-3.

32. Holewijn S, Den Heijer M, Swinkels DW, Stalenhoef AF, de Graaf J, Apolipoprotein B. non-HDL cholesterol and LDL cholesterol for identifying individuals at increased cardiovascular risk. J Intern 2010;268:567-77.

33. Denke MA. Weighing in before the fight: low-density lipoprotein cholesterol and non-high-density lipoprotein cholesterol versus apolipoprotein $\mathrm{B}$ as the best predictor for coronary heart disease and the best measure of therapy. Circulation 2005;112:3368-70.

34. Eliasson B, Cederholm J, Eeg-Olofsson K, Svensson AM, Zethelius B, Gudbjörnsdottir S. National Diabetes Register: clinical usefulness of different lipid measures for prediction of coronary heart disease in type 2 diabetes: a report from the Swedish National Diabetes Register. Diabetes Care 2011; 34:2095-100.

35. Kim SW, Jee JH, Kim HJ, Jin SM, SuhS, Bae JC, et al. NonHDL-cholesterol/HDL-cholesterol is a better predictor of metabolic syndrome and insulin resistance than apolipoprotein B/ apolipoprotein A1. Int J Cardiol. 2013;168:2678-83.

36. Zhao W, Gong W, Wu N, Li Y, Ye K, Lu B, et al. Association of lipid profiles and the ratios with arterial stiffness in middle-aged and elderly Chinese. Lipids Health Dis 2014;13:37.

37. Barter P, Gotto AM, LaRosa JC, Maroni J, Szarek M, Grundy SM, et al. HDL cholesterol, very low levels of LDL cholesterol, and cardiovascular events. N Engl J Med. 2007;357:1301-10.

38. Kinosian B, Glick H, Garland G. Cholesterol and coronary heart disease: predicting risks by levels and ratios. Ann Intern Med 1994;121:641-7.

39. Stampfer MJ, Sacks FM, Salvini S, Willett WC, Hennekens CH. A prospective study of cholesterol, apolipoproteins, and the risk of myocardial infarction. N Engl J Med. 1991;325:373-81.

40. Ridker PM, Stampfer MJ, Rifai N. Novel risk factors for systemic atherosclerosis: a comparison of C-reactive protein, fibrinogen, homocysteine, lipoprotein(a), and standard cholesterol screening as predictors of peripheral arterial disease. JAMA 2001;285:2481-5.

41. Frohlich J, Dobiasova M. Fractional esterification rate of cholesterol and ratio of triglycerides to HDL-cholesterol are powerful predictors of positive findings on coronary angiography. Clin Chem. 2003;49:1873-80.

42. Chiang JK, Lai NS, Chang JK, Koo M. Predicting insulin resistance using the triglyceride-to-high-density lipoprotein cholesterol ratio in Taiwanese adults. Cardiovasc Diabetol 2011;10:93.

43. Di Bonito P, Moio N, Scilla C, Cavuto L, Sibilio G, Sanguigno E, et al. Usefulness of the high triglyceride-to-HDL cholesterol ratio to identify cardiometabolic risk factors and preclinical signs of organ damage in outpatient children. Diabetes Care 2012;35:158-62.

44. Lesiewska H, Malukiewicz G, Mańkowska-Cyl A. OdrowążSypniewska G. Lipids and C-reactive protein as vascular risk markers in pseudoexfoliation syndrome. Acta Ophthalmol 2016;94:e380-e381.

45. Lesiewska H, Łukaszewska-Smyk A, Odrowąż-Sypniewska G, Krintus M, Mańkowska-Cyl A, Malukiewicz G. Chosen vascular risk markers in pseudoexfoliation syndrome: an age-related disorder. J Ophthalmol 2017;2017:5231095.

46. Türkyılmaz K, Oner V, Kırbas A, Sevim MS, Sekeryapan B, Özgür G, et al. Serum YKL-40 levels as a novel marker of inflammation and endothelial dysfunction in patients with pseudoexfoliation syndrome. Eye 2013;27:854-9.

47. Kurtul BE, Kurtul A, Ozer PA, Kabatas EU, Ertugrul GT. Serum lipid levels in pseudoexfoliation syndrome. Semin Ophthalmol 2017;32:281-4.

48. Spečkauskas M, Tamošiūnas A, Jašinskas V. Association of ocular pseudoexfoliation syndrome with ischaemic heart disease, arterial hypertension and diabetes mellitus. Acta Ophthalmol 2012;90:e470-e475.

49. Scicchitano P, Cameli M, Maiello M, Modesti PA, Muiesan ML, Novo $\mathrm{S}$, et al. Nutraceuticals and dyslipidaemia: beyond the common therapeutics. J Funct Foods. 2014;6:11-32. 